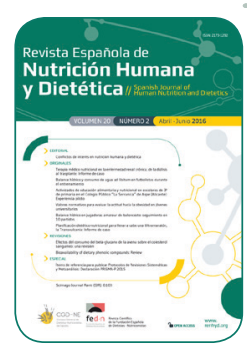

www.renhyd.org

\title{
EDITORIAL
}

\section{Conflictos de interés en nutrición humana y dietética}

\author{
Eduard Baladia $^{a, b, *}$, Rodrigo Martínez-Rodríguez ${ }^{a, b}$ \\ a Centro de Análisis de la Evidencia Científica de la Fundación Española de Dietistas-Nutricionistas (CAEC-FEDN), España. \\ b Red de Nutrición Basada en la Evidencia (RED-NuBE), España. \\ *e.baladia@rednube.net
}

Recibido el 27 de junio de 2016; aceptado el 28 de junio de 2016.

\section{CITA}

Baladia E, Martínez-Rodríguez R. Conflictos de interés en nutrición humana y dietética. Rev Esp Nutr Hum Diet. 2016; 20(2): 77 - 79 . doi: 10.14306/renhyd.20.2.261

A menudo los científicos tienden a pensar que existen conflictos de interés cuando hay intereses financieros en una investigación, sin embargo lo cierto es que la definición o el concepto de conflicto de interés es mucho más amplia.

Quizás el origen de esta interpretación pueda explicarse por la definición que Dennis F. Thomson ofreció del concepto en 1993 en The New England Journal of Medicine1: "Un conflicto de intereses es un conjunto de condiciones en la que el juicio profesional en relación a un interés primario (como por ejemplo el bienestar de un paciente o la validez e integridad de una investigación), tiende a ser indebidamente influenciado por un interés secundario (como por ejemplo el beneficio económico)". A pesar de la expresa mención de los conflictos de interés financieros en el título de la publicación y en la ejemplificación, la lectura completa del artículo identifica claramente otros intereses secundarios que pueden representar un conflicto de interés, tales como el reconocimiento científico, el avance en la carrera académica, o el éxito en la publicación de artículos ${ }^{1}$.
Sea como fuere, es un hecho constatado que los conflictos de interés (financieros o de otro tipo) tienen el potencial de producir sesgos importantes en la literatura científica ${ }^{2}$. Una revisión sistemática publicada en la revista JAMA en 2003 concluyó, tras revisar 8 artículos de revisión que evaluaban un total de 1.140 investigaciones, que existe una asociación positiva y estadísticamente significativa entre la financiación por parte de la industria y las conclusiones que favorecen a la misma (odds ratio 3,60; intervalo de confianza 95\%: 2,63-4,91)3. En la misma línea, una revisión Cochrane publicada en $2012^{4}$ concluyó, tras evaluar 48 artículos, que los estudios financiados por la industria obtienen más a menudo resultados favorables en términos de eficacia (riesgo relativo 1,24; intervalo de confianza 95\%: 1,14-1,35), de efectos adversos (riesgo relativo 1,87; intervalo de confianza $95 \%$ : 1,54-2,27), y en sus conclusiones (riesgo relativo 1,31; intervalo de confianza 95\%: 1,20-1,44).

Por si algún lector se ve tentado a pensar que este tipo de sesgos solamente ocurren por influencia de la industria del "Big Pharma", Michel Lucas y Elizabeth Loder lo expusieron 
de forma magistral y sencilla en sendas editoriales publicadas ambas en 2015: el "Big Food" también está sesgando las conclusiones de las investigaciones que financia ${ }^{5,6}$. En los editoriales se citan extensos trabajos de revisión como el realizado por Lesser y Cols. en $2007^{7}$ en el que se halló un odds ratio de 7,61 (intervalo de confianza 95\%: 1,27-45,73) de conclusiones favorables versus no favorables en las investigaciones relacionadas con la nutrición humana y financiadas por la industria en comparación a las no financiadas. Asimismo también se citan heroicos trabajos de establecimiento de influencias (financieras y de otros tipos, como de exitosa carrera académica) entre industria e investigadores, especialmente aquellas relacionadas con el "Big Sugar", el "Big Alcohol"9 y otras grandes compañías como las interesadas en los suplementos de calcio y de vitamina $D$ para la prevención de osteoporosis ${ }^{10}$, que también ha recibido la atención de este tipo de análisis. De dichas evaluaciones se pueden extraer algunas conclusiones escalofriantes: estas grandes compañías tienen la capacidad para influir en las conclusiones de grandes investigaciones, en ponencias científicas en congresos de alto impacto, en políticas y programas nutricionales gubernamentales y privados, en campañas de marketing dirigidas a niños/as, y, en definitiva, tienen capacidad de sesgar e influenciar de forma sustanciosa la práctica a nivel clínico pero también a nivel de salud pública ${ }^{11}$.

¿Y qué se puede hacer? ¿Los metaanálisis no son más neutros en este sentido? Pues desgraciadamente las revisiones sistemáticas y metaanálisis también se han visto afectadas por los conflictos de interés. Maira Bes-Rastrollo, de la Universidad de Navarra publicó en 2013 una revisión sistemática de revisiones sistemáticas sobre la influencia de la financiación de dichas revisiones sobre sus conclusiones en torno a la asociación del consumo de bebidas azucaradas en la ganancia de peso ${ }^{12}$, arrojando las siguientes conclusiones: las revisiones sistemáticas financiadas por la industria son cinco veces más proclives a presentar conclusiones de no asociación entre consumo de bebidas azucaradas y aumento de peso corporal (riesgo relativo: 5,0; intervalo de confianza $95 \%: 1,3-19,3)^{12}$. Pero seguro que el análisis de sesgos de 6 dominios de Cochrane es capaz de detectar los sesgos por conflictos de interés ¿no? Pues en realidad el sistema de Cochrane evalúa solamente los sesgos de: generación de secuencia aleatoria (sesgo de selección), ocultación de la asignación (sesgo de selección), cegado participantes-personal (sesgo de ejecución), cegado evaluadores (sesgo de detección), abandonos/pérdidas (sesgo de desgaste) e información selectiva (sesgo de información) ${ }^{13}$. De hecho en 2013 se publicó en The Cochrane Database of Systematic Reviews un artículo en el que se pedía formalmente la inclusión sistemática del análisis de los conflictos de interés en la herramienta de evaluación de sesgos de la organización ${ }^{14}$.

¿Y eso también me afecta a mí? Una revisión sistemática publicada en 2013 en Plos Medicine puso de relieve que las interacciones entre industria y profesional afectan también a profesionales sanitarios no médicos ${ }^{15}$. En dicha revisión sistemática se citan de forma específica a los/las dietistas, los cuales mantienen, a pesar de ser conscientes de los problemas relacionados con los sesgos en las investigaciones, actitudes positivas hacia la interacción con la industria, denominándola a veces de forma cariñosa como "el diablo necesario"15. A pesar de que la interacción industria-profesional no es sinónimo de mala praxis en investigación o en la práctica clínica, los datos sobre su influencia en las conclusiones de los estudios son claros y contribuyen a la percepción de que "la ciencia de la nutrición humana y dietética está en venta" ${ }^{\prime \prime}$. En palabras de Young2: "No siempre somos conscientes de nuestros propios sesgos. La idea de que los científicos son buscadores de la verdad objetiva es una ficción agradable, pero contraproducente en la medida en que puede disminuir la vigilancia contra el sesgo". Ante este problema, autores/as, revisores/as y editores/as, deben involucrarse y tomar parte en su manejo ${ }^{2}$, declarar tus conflictos de interés (financieros o de otro tipo) de forma adecuada y detallada es una obligación de todos/as.

\section{Conflicto de intereses}

EB y RM son editores de la Revista Española de Nutrición Humana y Dietética y miembros del Centro de Análisis de la Evidencia Científica y de la Red de Nutrición Basada en la Evidencia, los cuales, pese a no ser financiados directamente por ninguna industria alimentaria, son propiedad de la Fundación Española de Dietistas-Nutricionistas (FEDN), quien mantiene convenios de colaboración con la industria alimentaria que implican contraprestación económica. RM, además, trabaja para una empresa privada del sector alimentario y recibe un salario por ello. En el presente editorial se han ofrecido de forma deliberada ejemplos y datos que no favorecen a los intereses de la industria alimentaria con la que colabora la FEDN como muestra del nivel de independencia científica de la que goza la presente revista científica y los mencionados proyectos.

\section{REFERENCIAS}

(1) Thompson DF. Understanding financial conflicts of interest. N Engl J Med. 1993; 329(8): 573-6.

(2) Young SN. Bias in the research literature and conflict of interest: an issue for publishers, editors, reviewers and authors, and it is not just about the money. J Psychiatry Neurosci JPN. 2009; 34(6): 412-7. 
(3) Bekelman JE, Li Y, Gross CP. Scope and impact of financial conflicts of interest in biomedical research: a systematic review. JAMA. 2003; 289(4): 454-65.

(4) Lundh A, Sismondo S, Lexchin J, Busuioc OA, Bero L. Industry sponsorship and research outcome. Cochrane Database Syst Rev. 2012; 12: MR000033.

(5) Lucas M. Conflicts of interest in nutritional sciences: The forgotten bias in meta-analysis. World J Methodol. 2015; 5(4): 175-8

(6) Loder E. Big food, big pharma: is science for sale? BM]. 2015; 350: h795.

(7) Lesser LI, Ebbeling CB, Goozner M, Wypij D, Ludwig DS. Relationship between funding source and conclusion among nutrition-related scientific articles. PLoS Med. 2007; 4(1): e5.

(8) Gornall J. Sugar: spinning a web of influence. BM]. 2015; 350: h231.

(9) Editors TPIM. Let's Be Straight Up about the Alcohol Industry. PLOS Med. 2011; 8(5): e1001041.

(10) Grey A, Bolland M. Web of industry, advocacy, and academia in the management of osteoporosis. BM]. 2015; 351: h3170.
(11) Editors TPIM. PLoS Medicine Series on Big Food: The Food Industry Is Ripe for Scrutiny. PLOS Med. 2012; 9(6): e1001246.

(12) Bes-Rastrollo M, Schulze MB, Ruiz-Canela M, MartinezGonzalez MA. Financial conflicts of interest and reporting bias regarding the association between sugar-sweetened beverages and weight gain: a systematic review of systematic reviews. PLoS Med. 2013; 10(12): e1001578; dicsussion e1001578.

(13) Higgins JPT, Altman DG, Sterne JAC (editors). Chapter 8: Assessing risk of bias in included studies. In: Higgins JPT, Green S (editors). Cochrane Handbook for Systematic Reviews of Interventions Version 5.1.0 (updated March 2011). The Cochrane Collaboration, 2011. Available from: www.handbook.cochrane.org

(14) Bero LA. Why the Cochrane risk of bias tool should include funding source as a standard item. Cochrane Database Syst Rev. 2013; (12): ED000075.

(15) Grundy Q, Bero L, Malone R. Interactions between nonphysician clinicians and industry: a systematic review. PLoS Med. 2013; 10(11): e1001561. 